\title{
A Critical Review of Sentiment Analysis
}

\author{
Fatehjeet Kaur Chopra \\ Punjabi University Regional Centre for Information \\ Technology and Management, \\ Mohali, Punjab, India
}

\author{
Rekha Bhatia, PhD \\ Punjabi University Regional Centre for Information \\ Technology and Management, \\ Mohali, Punjab, India
}

\begin{abstract}
Sentiment analysis is the process which helps in recognizing people's perspective and emotional conditions. Sentiment analysis appeared in the roots of the disciplines of psychology, sociology and anthropology. Sentiment analysis takes place from the hypothesis of emotive attitude and assessment hypothesis. It emphasis on sensation in forming perceptions. Feelings that are generated from both conscious and unconscious processing are called emotions. The feelings of the people can be expressed in positive or negative ways. Mostly, parts of speech are used as feature to extract the sentiment of the text. Sentiment analysis is an evolving field with a variety of use applications. Further, the evaluation of the accuracy of the existing systems, from which it is analyzed that the result can be improved by calculating the sentiments of word instead of calculating sentiment of complete sentence or paragraph.
\end{abstract}

\section{Keywords}

Sentiment Analysis, Opinion mining, NLP, Linguistic Resources, Web data Analysis, Information Retrieval

\section{INTRODUCTION}

Sentiment Analysis administers inspecting feelings, sentiments, and the mentality of a speaker or an author from a given portion of text. "Sentiment analysis (opinion mining) refers the utilization of custom unrefined (natural) language processing, computational semantics and satisfied inquiring to differentiate and withdraw internal facts in reference matters".

Sentiment analysis (opinion mining) is a multidisciplinary and multifaceted artificial intelligence issue. Its point is to minimize the hole in the middle of human and PC. In this way, it is gathering of human insight and electronic knowledge for mining the content and arranging client notions, likes, despises and wishes [1].

Sentiment analysis(opinion mining) includes categorizing beliefs in words into classes same as "positive" or "negative" frequently accompanied by an implied class of "neutral". Opinion mining or power of speech of the customer is other names of Sentiment Analysis. Viewpoint(s) unexpressed by a text span is attempted to be identified by Sentiment analysis; for example an application that classifies a movie review as "thumbs up" or "thumbs down". A novel machine-learning method is proposed that applies text-categorization methods to just the subjective portions of the document for determining the sentiment polarity(for classification).Techniques for finding minimum cuts in graphs can be used to implement extraction of these portions and by this incorporation of crosssentence contextual constraints is facilitated greatly.

Three main classification levels in Sentiment Analysis which are document-level, sentence-level, and aspect-level Opinion mining(Sentiment Analysis).

\subsection{Document-level}

Instructing of an evaluation report as conveying a positive or negative emotion or slant is expected by Sentiment Analysis. A fundamental data unit is considered as an entire archive.

\subsection{Sentence-level}

Group conclusion to be communicated in every sentence is intended by Sentiment Analysis. Distinguishing whether the sentence is subjective or objective is the principle step. When the sentence is subjective, if the sentence communicates positive or negative assessments is figured out by Sentencelevel SA. Number of applications and upgrades on SA calculations were proposed in the most recent couple of years. A more critical look on these improvements is intended to be given by this overview and outlined and arranged a few articles displayed in this field which are indicated by the different SA strategies. The writers have gathered fifty-four articles which exhibited critical upgrades to the SA field recently. A wide mixed bag of Sentiment Analysis fields is covered by these articles.

Market research well knows Sentiment Analysis. Utilizing out of date consumer remark cards, researches, evaluations and center sections, views or opinions have been examined through the market. For proceeding benefits of the internet mutual habitat some tools can be adapted, but only researcher presence and small sample sizes can use them. By systematic collection and analyzation of online sentiments through a large sample of customers in real time, these problems can be addressed by Sentiment analysis. Online sentiments are conceptualized as human convictions or emotions expressed on the internet. With the most popular social network sites, attitude is usually expressed from various media alternatives towards a situation, event or object.

Online sentiments examples are:

"I actually love my new Smart phone"

"This picture is the ultimate picture I have ever seen"

"Taste of bagels is worse in town"

So systematic analysis of online expressions is known as Sentiment analysis. Sentiment analysis (opinion mining) emphasis on viewpoint assessment and views of beliefs on a subject of regard utilizing mechanism studying approaches particularly.

\section{LITERATURE REVIEW}

(Jeonghee Yi, 2003) [2] has stated that extraction of sentiment (or opinion) about a subject from online text documents is done by sentiment Analyzer (SA). All references to the given subject is detected by SA, and sentiment in each references using natural language processing (NLP) techniques is also determined by SA, instead of classifying the sentiment of an entire document about a subject. In this sentiment analysis has 1) A topic specific feature term extraction, 2) Sentiment extraction, and 3) Subject, sentiment association by 
relationship analysis. Sentiment Analysis for the analysis utilizes two linguistic resources :the sentiment lexicon and the sentiment pattern database. Online product review articles ("digital camera" and "music" evaluation(reviews)), and general documents (web pages and news article also included ) verifies the performance of the algorithms.

(Pang B, Opinion mining and sentiment analysis., 2008) [3] have analyzed concept in (Liu, 2004); the academics have undertaken a diverse range of related research, due to its practicality in opinion monitoring and business competitive intelligence. Sentiment analysis on online reviews has become increasingly popular. A multidisciplinary research field in nature, various areas like natural language processing (NLP), computational language rules, information recovery, machine intelligence and artificial intelligence etc are included in sentiment analysis. No research on the commercial value of online product reviews.

(Wilson T, 2005) [4] have pointed out that nature of sentiment expressions are not necessarily subjective. As sentences are just short documents so no basic distinction in the middle of document and sentence level classes is there (B L., 2012). Document level or sentence level classes of wordings does not supply or give the required piece of information needed; so there occurs a necessity to go to the aspect level. Categorizing the view or emotion with respect to the particular features of bodies is the focus or point of the Aspect-level SA. Recognizing the bodies and their features is the main first step. Dissimilar beliefs for dissimilar features of the identical body can be stated by belief possessors same as sentence "The battery life is extensive, but voice quality of this phone is not good". The first two types of Sentiment Analysis can be approached by this observation. A chief matter in this area is the data sets that are used in SA. From product reviews the main sources of data can be taken. Sentiment Analysis (SA) is not only implemented on product assessment but can also be implemented on stock markets (Yu Liang-Chih, 2013)[5] and (Michael Hagenau, 2013)[6], news reports(articles), (Tao Xu, 2012) [7] or political discussions (Maks Isa, 2012)[8]. For example, people's beliefs on a definite election applicants or political parties could be figured out in political debates. Prediction of election results can also be done from political posts. In the SA process, they are also used as data sources.

As most famous SA techniques and applications are covered in one research paper, so in this field for new comer researchers this survey can be useful. A purified classification to the different Sentiment Analysis (SA) methods which is not originated in other observations is given uniquely by this survey. New associated areas in Sentiment Analysis (SA) which have allured the researchers recently and their correlated papers(reports) are discussed. In these areas Emotion Detection (ED), Building Resources (BR) and Transfer Learning (TL) are incorporated .Extraction and analyzation of emotions is the goal of emotion detection, while in the sentences the feelings could be unreserved (direct) or indirect. Transfer studying (or learning) or Cross-Domain categorization is concerned with inspecting statistics from one area and then utilizing the outcomes in a objective field. .Creating lexica, corpora are the goal of building resources in which opinion expressions are elucidated in proportion to their polarity, and sometimes glossaries. Every year in the SA field's great number of articles are introduced. Along years number of articles is growing. By this the requirement to have observation documents have been created that abstract the latest analysis styles and ways of Sentiment Analysis(SA).Some worldly-wise and complete surveys can be found by the reader including (Tsytsarau
Mikalai, 2012)[9], (B L. , 2012)[10], (Pang B, Opinion mining and sentiment analysis, 2008)[3] , (Cambria E, 2013)[11], (Feldman, 2013)[12] and (Montoyo Andre' s, 2012;)[13]. The issue of Sentiment Analysis (SA) from the execution viewpoint not from the Sentiment Analysis(SA) methods viewpoint have been reviewed by those surveys.

(Yu Liang-Chih, 2013) [5] Has shown their research that extraction of people's opinions on features of an entity is the important task of opinion mining. There is a need to assemble these words and phrases, which are domain synonyms, into the same feature group to produce a useful synopsis, . Also, there is a complication in the sentiment relation of the features and opinions .To deal with the feature-level opinion mining problems, a novel method is proposed.

1) The explicit features and the implicit features are contemplated in the proposed method.

2) Vague opinion words and clear opinion words are the categorized divisions of opinion words, which aim to discover the implicit features and clutch the features. There are three aspects on which clutching of features depend: The correlated opinion words, the resemblance of the features and the formation of the features.

Also, to strengthen the clutching in the procedure the context information is used, which is shown to be useful in clustering or clutching. Feature-level opinion mining including three steps: (1) Extract the features and the corresponding opinions (2) Cluster the features (3) Orient the opinions of the feature.

Feature extraction for entities is an important task for opinion mining. This paper proposed a new method to deal with this problem. The new method uses the corresponding opinion words extracting the features, and according to mutual support and confidence to filter the noise. It also identifies the implicit features and clusters the features based on the knowledge of the background which strengthen cluster results. Empirical evaluation show the proposed method outperforms. However, this method has some shortcomings. Small scale corpus cannot (Yao \& Chen, 2013) [14] perform well. And the structure of the vague opinions dictionary and part-of-speech dictionary increases the cost of the method. Next, the establishment of two dictionaries by automatically and improve the precision and recall for the small scale corpus have been studied.

(Singh \& Piryani, 2013) [15] has stated an fact-finding effort work on a new type of field particular feature-based investigatory for aspect-level sentiment analysis of picture analysis(reviews). A feature directed plan that examines the textual assessment of a picture have been conceived and sentiment tag on each point is allocated to it. The scores on each side or point from various analyses are then summated and a final description of the picture is created on all boundaries. A Senti Word Net established plan with two dissimilar semantic attribute choices consisting of adjectives, adverbs and verbs and n-gram attribute withdrawal is utilized. Also a Senti Word Net plan is utilized to calculate the document-level sentiment for each picture inspected and contrasted the outcomes with outcomes acquired utilizing Alchemy API. The sentiment description of a picture is also contrasted with the document-level sentiment outcome. The outcomes acquired show that the plan provides a more precise and concentrated sentiment description than the uncomplicated document-level sentiment analysis.

There are mainly three kinds of viewpoints for sentiment categorization of words: 
(a) Machine learning based text classifier like Naïve Bayes, SVM or KNN- with satisfactory attribute selection plan are used;

(b) Unrestricted semantic alignment plan of extricating applicable n-grams of the words (text) are used and then tagging them either as positive or negative and considerably the report or document; and

(c) Senti Word Net rooted publicly obtainable library that gives positive, negative and neutral scores for words or texts is used. Some of the applicable previous works on sentiment categorization can be originated in (K. Dave, 2003)[16], (Turney P. D., 2002)[17], (F.Sebastiani, 2005)[18].

(Varghese, 2004) [19] has used the concept where the word level extent attribute extraction is done utilizing Naive Bayesian Classifier. The semantic alignment of the separate sentences is recovered from the contextual data or information. This machine learning viewpoint on average normally affirms an precision rate of $83 \%$. Another remarkable work is the execution of both Natural Language perception and creation in Sentiment analysis (SA) (Pang B, Opinion mining and sentiment analysis, 2008).

\section{OBJECTIVES}

Sentiment analysis is an emerging research field and this task is very important because peoples spent their most of the time on the web. In this an approach is proposed to determine the sentiment orientation i.e. polarity of the Punjabi general evaluation (reviews)s by scoring procedure. Sentiment analysis (SA) is needed to be performed in Punjabi language because of the growth in Punjabi facts or figures on the web. Separate positive and negative summarized results are created which is useful for the user in making the decision. The objective of this is

- To Study the algorithm of existing sentiment analysis in various non-identical languages like Hindi, Punjabi and English.

- To make an application that maintains the directory of positive, negative and neutral words corresponding to their values.

- To implement an algorithm that analyzes the sentiments of entered Punjabi paragraph and highlights the words according to their sentiment.

- Algorithm calculates the result according to value of highlighted words.

- To find the results of proposed algorithm with the help of negative, positive and neutral words database maintained in the application.

\section{CONCLUSION}

Sentiment analysis appeal is tremendously developing. The researchers can be faced with problems, oppositions and challenges; though semantic analysis is emerging but this field is still new to them .Problem related to the nature of classification is a possible challenge. Classification techniques that create two or three groups at most, there is a limit in the extraction of groups and subgroups. Text based data that is valid in specific places at specific times are usually context specific and domain dependent. Sentiment observation has a broad type of approaches in information systems, which includes categorizing reviews, encapsulating analysis (review) and different real time applications. There are probably to be various distinct applications particularly not examined. It is constituted that sentiment words (classifiers) are acutely based on fields or areas or topics. In time to come, more work is required on furthermore bettering the performance evaluations. Sentiment analysis can be tried or auditioned for new applications. Even though the algorithms and methods used for Sentiment analysis (SA) are proceeding fast, but, a lot of issues in this area of study prevail unsolved. The main demanding details exist in hold of other languages, trading with negation proclamations(expressions); construct or yield a summary of beliefs build on product features(attributes), difficulty of sentence(document), managing of implied(indirect) product attributes etc. More research could be committed to these problems (challenges) in time to come.

\section{REFERENCES}

[1] Kaur, A., \& Gupta, V. (Nov. 2013). A survey on sentiment analysis and opinion mining techniques. Proceedings of Journal of Emerging Technologies in Web Intelligence, Vol.5, No. 4. 62.

[2] Jeonghee Yi, T. N. (2003). "Sentiment Analyzer: Extracting Sentiments about a Given Topic using Natural Language Processing Techniques". Proceedings of the Third IEEE International Conference on Data Mining . IEEE.

[3] Pang B, L. L. (2008). Opinion mining and sentiment analysis. Foundations and trends in information retrieval , 1-2 (2), 1-135.

[4] Wilson T, W. J. (2005). Recognizing contextual polarity. In: Proceedings of HLT/EMNLP.

[5] Yu Liang-Chih, W. J.-L.-C. (2013). Using a contextual entropy model to expand emotion words and their intensity for the sentiment classification of stock. KnowlBased Syst, (pp. 41:89-97).

[6] Michael Hagenau, M. L. (2013). Automated news reading: stock price prediction based on financial news using context-capturing features. Decis Supp Syst.

[7] Tao Xu, P. Q. (2012). Identifying the semantic orientation of terms using S-HAL for sentiment analysis. (pp. 35:279-89). Knowl-Based Syst .

[8] Maks Isa, V. P. (2012). A lexicon model for deep sentiment analysis and opinion mining applications. (pp. 53:680-8). Decis Support Syst.

[9] Tsytsarau Mikalai, P. T. (2012). Survey on mining subjective data on the web. (pp. 24:478-514). Data Min Knowl Discov. 64

[10] B, L. (2012). Sentiment analysis and opinion mining. Synth Lect.

[11] Cambria E, S. B. (2013). New avenues in opinion mining and sentiment analysis. IEEE Intell Syst (pp. 28:15-21). IEEE.

[12] Feldman, R. (2013). Techniques and applications for sentiment analysis. Commun ACM , (pp. 56:82-9).

[13] Montoyo Andre' s, M.-B. P. (2012;). Subjectivity and sentiment analysis: an overview of the current state of the area and envisaged developments. (pp. 53:675-9). Decis Support Syst.

[14] Yao, R., \& Chen, J. (2013). Predicting Movie Sales Revenue using Online Reviews. International Conference on Granular Computing (GrC). IEEE. 
[15] Singh, V., \& Piryani, R. (2013). Sentiment Analysis of Movie Reviews A new feature -based heuristic for aspect-level sentiment classification. IEEE.

[16] K. Dave, S. L. (2003). "Mining the Peanut GalleryOpinion Extraction and Semantic Classification of Product Reviews". Proceedings of the 12th International World Wide Web Conference, (pp. 519-528).

[17] Turney, P. D. (2002). Thumbs up or thumbs down? Semantic orientation applied to unsupervised classification of reviews. Proc. 40th Annual Meeting on Association for Computational Linguistics (ACL), Philadelphia , US.
[18] F.Sebastiani, A. E. (2005). "Determining the Semantic Orientation of terms through gloss analysis". Proceedings of CIKM-05, 14th ACM International Conference on Information and Knowledge Management (pp. 617-624,). Bremen, DE, CIKM.

[19] Varghese, R. (2004). Opinion Mining Based on FeatureLevel Aspect Based Sentiment Analysis using Support Vector Machine Classifier. International Conference on Advances in Computing, Communications and Informatics (ICACCI),ICACCI.Heba Khudhair Abass(2013), “A Study of Digital Image Fusion Techniques Based on Contrast and Correlation Measures". 